\title{
REDES OPTIMAS CON COSTOS ACUMULATIVOS Y COMUNICACIÓN BIDIRECCIONAL
}

Juan M.C. Larrosa*

Fernando A. Tohmé*

\begin{abstract}
Resumen
Este trabajo es una extensión al enfoque de Larrosa y Tohmé (2003) en el cual se modifica la función de pagos al permitir que la información fluya en ambos sentidos. Los costos siguen siendo pagados por quién inicia la conexión por lo que esta asimetría revela cambios en la topología final de equilibrio. Encontramos que diversas topologías óptimas persisten como redes de Nash pero que una red estricta de Nash se corresponde con la red de línea secuencial con nodos activos intermedios, es decir, los agentes se colocan en una línea y, agente de por medio, se van conectando con el agente predecesor y con el agente posterior. De ese modo se interrumpe la acumulación de costos y los beneficios son maximizados.
\end{abstract}

Clasificación JEL: C72, L13, L20

Palabras clave: juegos de formación de redes - comunicación bidireccional - red de línea

\begin{abstract}
This work is an extension of Larrosa y Tohmé (2003)'s approach only differing in the agents' payoff function. This modification allows that information flows in both ways. Costs still are paid for who initiates the connection and this asymmetry reveals changes in the equilibrium topology. We find several optimal topologies as Nash equilibrium but only the sequential linear network with intermediate activation nodes as strict Nash equilibrium, i.e., agents are set in line and every intermediate agent connect with her two immediate neighbors. This way, cost acummulation is interrupted and profits are maximized.
\end{abstract}

JEL Classification System: C72, L13, L20

Keywords: Network formation games - two-way flow communication - linear network

\section{INTRODUCION}

La interacción entre agentes puede ser representada en diversas formas. Una manera de representar los intercambios directos que ha atraído mucho interés en los últimos años es a través de las redes. Dado que es fácil de entender, esta herramienta de análisis fue primera-

\footnotetext{
* Departamento de Economía, Universidad Nacional del Sur, Consejo Nacional de Investigaciones Cientificas y Técnicas (CONICET) juflarrosa@ criba.edu.ar, fthome@ criba.edu.ar
} 
mente adoptada en la sociología y antropología. Para los expertos de esas áreas constituye una forma pictórica de entender la influencia de los vecindarios de agentes sobre las conductas individuales. Enfocados en las redes sociales reales, los sociólogos y antropólogos han acumulado una vasta cantidad de evidencia que ayuda a entender como el comportamiento humano es moldeado por el comportamiento de otros agentes.

En términos matemáticos, una red es un grafo, donde los nodos representan agentes individuales y los arcos o enlaces representan el bien de utilidad (por ejemplo, información, prestigio personal, etc.) que es intercambiado. La literatura económica introdujo recientemente herramientas de teoría de juegos en este marco de análisis. En vez de estar interesados en sólo los aspectos descriptivos, algunos teóricos de la economía han encarado el estudio de cómo las redes se forman, en primer término, y qué las hace estables o eficientes (Jackson y Wolinsky 1996, Bala y Goyal 2000, Dutta y Jackson 2000). El enfoque de redes basado en teoría de juegos exhibe dos corrientes principales: una basada en juegos cooperativos y otra con fuerte sentido estratégico. El análisis basado en juegos cooperativos, como es usual en este enfoque, estudia el problema de formación de coaliciones entre agentes. El duro supuesto de transferencia de utilidad entre agentes es difícil de justificar en muchos casos, así como muchas veces computacionalmente costoso (Qin 1996, Dutta y otros 1998, Slikker y van den Nouweland 2001).

El enfoque estratégico o no cooperativo, a su vez, solo requiere la definición de estrategias disponibles para los agentes así como la caracterización de las correspondientes funciones de pagos. Dado cierto protocolo o regla de interacción, los agentes deciden sí se conectan o no la red, evaluando los beneficios de conexión (o desconexión) con otros agentes. Las decisiones racionales de los agentes llevan a equilibrios de Nash, las cuales sostienen las redes que son el foco de este esquema de análisis. Esto es, precisamente, el enfoque adoptado por este trabajo.

Este trabajo sigue la línea de Larrosa y Tohmé (2003) aunque ampliando ahora la posibilidad de circulación de la información. Consideramos que la información fluye bidireccionalmente mientras que los enlaces son sostenidos por quienes inician los enlaces. Bajo este esquema de pagos, la función de costos es acumulativa. A medida que la información pasa por más agentes se hace más cara ¿Qué topología óptima de comunicación soporta estas condiciones? Encontramos que un tipo de red lineal secuencial es la que permite el mínimo de conexiones con el máximo flujo de información.

En la sección I comienza el análisis con la definición de grafos de flujo bidireccional. La sección II se observa la presentación del modelo. En la sección III se desarrolla la arquitectura de equilibrio así como la forma en que equilibrios verifican algunos criterios de estabilidad y optimalidad. La sección IV discute las analogías y diferencias con el caso monodireccional y concluye el trabajo.

\section{I .EL CASO DE FLUJO DE INFORMACION BIDIRECCIONAL}

Se modelarán las redes como grafos dirigidos con flujos bidireccionales. El ageInte estableciendo enlaces con otros agentes puede tener su información pero los otros acceden a la información poseída por ella automáticamente. Quién inicia el enlace es quién debe pagarlo. 
Esta asimetría modificará las estructuras óptimas respecto al caso considerado cuando el flujo de información es monodireccional (Larrosa y Tohmé, 2003).

\section{I.1 Definiciones}

A continuación se definen conceptos y herramientas comunes al desarrollo de toda la argumentación del presente trabajo. Sea $N=(1, \ldots, n)$ un conjunto de agentes. Para evitar resultados triviales siempre vamos a suponer que $n \geq 3$. Si $i$ y $j$ son dos miembros típicos de $N$, un enlace entre ellos, sin intermediarios, originado en $i$ y finalizado en $j$ será representado como $i j$. La interpretación de $i j$ es que $i$ establece un contacto con $j$ que permite a $i$ tener acceso a la información de $j$ así como a su red de contactos.

Cada agente $i \in N$ tiene alguna información de su propiedad, $v_{i} \in Z_{+}$(es decir, representado como un entero positivo). Por convención inicialmente supondremos que la información de cada agente es lo suficientemente valiosa como para que valga la pena establecer un enlace con ella ${ }^{1}$, es decir que $v_{i}>1$. Como se mencionó $i$ puede acceder a más información formando enlaces con otros agentes. Los agentes tratarán de maximizar la utilidad de la información disponible para ellos así como minimizar el costo de conexión con otros agentes. Para lograr ello, ellos estarán dotados con un conjunto de estrategias. Cada estrategia para $i \in N$ es un vector $g_{i}=\left\langle g_{i, 1}, \ldots, g_{i, i-1}, g_{i, i+1}, \ldots, g_{i, n}\right\rangle$ de $(n-1)$ dimensiones donde cada $g_{i, j}$ para $j \neq i$ vale 0 o 1 . Esto es interpretado como que $i$ establece un enlace directo con $j$ si $g_{i, j}=1$ mientras que si $g_{i, j}=0$ dicho enlace no existe. El conjunto de todas las estrategias se anota como $G_{i}$. El análisis se restringe sólo a los casos de estrategias puras, lo cual implica que $\left|G_{i}\right|=2^{n-1}$. Finalmente, $G=G_{1} \times \ldots \times G_{n}$ denota el conjunto de perfiles de estrategia en la interacción entre los agentes de $N$.

Se entiende existe un sendero desde $j$ hacia $i$ de acuerdo al $g \in G$ si existe una secuencia de agentes diferentes (para evitar que existan ciclos) $j_{0}, \ldots, j_{m}$ (con $i=j_{0}$ y $j=j_{m}$ ) tal que $g_{j_{0}, j_{1}}=\ldots=g_{j_{m-1}, j_{m}}=1$. En palabras, dada una estrategia conjunta $g$, tal que $j_{1} \in N^{g_{j_{0}}}$, $j_{2} \in N^{g_{j_{1}}}, \ldots, j_{m} \in N^{g_{j_{m-1}}}$. Un sendero desde $j=j_{m}$ hacia $i=j_{0}$, denotada como $j \stackrel{g}{\rightarrow} i$, tiene una longitud, la cardinalidad de la secuencia $j_{1}, j_{2}, \ldots, j_{m-1}, j_{m}$, es decir $m$, el cual indica el número de enlaces intermedios entre $j$ e $i$. Nótese que un enlace dirigido es un sendero de longitud 1.

Otros supuestos del modelo son:

(i) El valor $v_{i}$ no cambia a medida que más jugadores conozcan dicha información;

(ii) El valor de $v_{\mathrm{i}}$ no cambia a medida que un individuo en particular conozca más información, $\mathrm{y}$

\footnotetext{
${ }^{1}$ Con este supuesto descartamos la posibilidad de la aparición de la red vacía (es decir, los agentes permanecen todos aislados) como estructura de equilibrio en nuestro modelo.
} 
(iii) Las informaciones son transmitidas claramente a través de la red (no hay distorsión de información ni decaimiento de flujo) tal que el valor de $v_{i}$ para $j$ es el mismo sea que $i$ y $j$ estén conectados directa o indirectamente.

A continuación se analiza el caso en que la información fluye en dos direcciones una vez establecido el contacto.

\section{EL MODELO}

En esta sección también se considera el caso de grafos dirigidos. Si bien la información fluye hacia ambos lados a través de un enlace (comunicación bidireccional, análoga a la observada en los grafos no dirigidos), estos grafos son dirigidos porque toman en cuenta el costo de iniciación del enlace ( $g_{i j}=1$ implica que $i$ financia el enlace y, por lo tanto, no tiene la misma interpretación que $g_{j i}=1$ tal como lo requiere la definición de grafo no dirigido). Aquí lo que se destaca es la simetría en los beneficios de quienes están conectados (ambos disfrutan de la misma información) y la asimetría de los costos (quién inicia el enlace debe solventar la conexión).

El problema es determinar cuál estructura puede emerger como equilibrio estratégico entre los agentes y si ésta es óptima o no. Se encuentra que los equilibrios estrictos de Nash o equilibrios de Nash con el mínimo número de enlaces sostienen una red de estrella periféricamente solventada, la cual es estable y óptima.

Vease un ejemplo análogo al Ejemplo 1 para el caso no dirigido.

Ejemplo 1. Considérese un grupo de cuatro agentes, $N=\{a, b, c, d\}$. Una estrategia conjunta $g=\left\langle g_{a}, g_{b}, g_{c}, g_{d}\right\rangle$ puede ser representada como un perfil de estrategias. Esto se puede ver en la Tabla 1 (el asterisco indica quién inició el enlace).

Cada fila es la estrategia elegida por uno de los agentes. Las columnas corresponden a los agentes. Una entrada 1 en la fila $i$ y columna $j$ significa que la estrategia del agente $i$ prescribe establecer un enlace con el agente $j$. Las entradas en la diagonal principal están con cru- 
ces dado que los agentes no pueden establecer un enlace con ellos mismos. En la Figura 8 puede verse el grafo dirigido que se corresponde con $g$.

Tabla 1- Perfil de estrategias para el Ejemplo 1

\begin{tabular}{|l|c|c|c|c|}
\hline Estrategia & $a$ & $b$ & $C$ & $d$ \\
\hline$g_{a}$ & $\mathrm{X}$ & $1^{*}$ & 0 & 0 \\
\hline$g_{b}$ & 1 & $\mathrm{X}$ & $1^{*}$ & 0 \\
\hline$g_{c}$ & 0 & 1 & $\mathrm{X}$ & $1^{*}$ \\
\hline$g_{d}$ & 0 & 0 & 1 & $\mathrm{X}$ \\
\hline
\end{tabular}

Figura 1- Red formada por el perfil de estrategias del Ejemplo 1

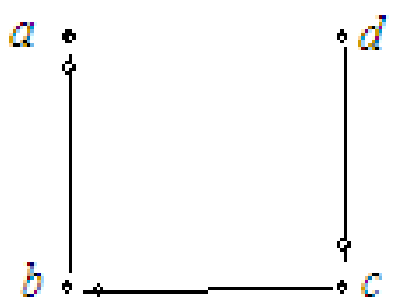

Siguiendo a Bala y Goyal (2000: 1190-1191) se denota la clausura de $g$ como $\bar{g}=\operatorname{cl}(g)$ y definida por $\bar{g}_{i, j}=\max \left\{g_{i, j}, g_{j, i}\right\}$ para cada $i$ y $j$ en $N$. Se define $N_{d}^{g_{i}}=\left\{k \in N \mid g_{i, k}=1\right\}$ como el conjunto de agentes con quiénes $i$ establece un enlace directo de acuerdo a su perfil de estrategias $g_{i}$. Se denota también a $N_{u}^{g_{i}}=\left\{h \neq k|h \in N| g_{i, h} \geq 2\right\}$ como el conjunto de agentes con quiénes $i$ establece un enlace indirecto de acuerdo a su perfil de estrategias $g_{i}$. Considérese $N^{g_{i}}(i ; g)=\{k \in N \mid i \stackrel{g}{\leftrightarrow} k\}$ como el conjunto de agentes con quiénes $i$ establece un enlace dirigido bidireccional de acuerdo a su perfil de estrategias $g_{i}$. Se considera que existe un sendero desde $j$ hacia $i$ de acuerdo al $g \in G$ si existe una secuencia de agentes diferentes (para evitar que existan ciclos) $j_{0}, \ldots, j_{m}$ (con $i=j_{0}$ y $j=j_{m}$ ) tal que $g_{j_{0}, j_{1}}=\ldots=g_{j_{m-1}, j_{m}}=1$. En palabras, dada una estrategia conjunta $g$, tenemos que $j_{1} \in N^{g_{j_{0}}}$, $j_{2} \in N^{g_{j_{1}}}, \ldots, j_{m} \in N^{g_{j_{m-1}}}$. Un sendero desde $j=j_{m}$ hacia $i=j_{0}$, denotada como $j \stackrel{g}{\leftrightarrow} i$, tiene una longitud, la cardinalidad de la secuencia $j_{1}, j_{2}, \ldots, j_{m-1}, j_{m}$, es decir $m$, el cual indica el número de enlaces intermedios entre $j$ e $i$. Nótese que un enlace dirigido es un sendero de longitud 1.

La existencia de un enlace directo $i j$ indica una comunicación simétrica entre $i$ y $j$. Esto es, $g_{i, j}=1$ indica que $i$ ha establecido una comunicación con $j$ que le permite acceder a la información de $j$ y pero también le permite a $j$ acceder a la información poseída por $i$. La asimetría surge en los costos. Aquí, $i$ ha iniciado la conexión por lo que es ella quién debe sol- 
ventar el costo de la conexión, mientras que $j$ no incurre en costo alguno. Las estructuras con esta característica son denominadas redes de flujo bidireccional.

En las redes de flujo bidireccional el perfil de estrategias puede ser representado como un grafo dirigido $g=\left(g_{1}, \ldots, g_{n}\right)$ sobre $N$. Esto es, en el grafo dirigido bidireccional los elementos de $N$ son los nodos mientras que cada enlace establecido como $g_{i, j}=1$ es representado por una línea empezando en $j$ con un círculo blanco sobre la línea a medida que se aproxima a $i$ (esto es para representar la idea que cuando $i$ fue quien inició el enlace; la información circula en ambas direcciones).

Se expresa al conjunto de agentes accedidos (directamente o de otro modo) por $i$ como $N^{i ; g}=\{k \in N \mid k \stackrel{g}{\rightarrow} i\}$. No se incluye a $i$ a fin de facilitar las derivaciones posteriores del modelo $^{2}$. Nótese que $N^{i ; g}=N_{d}^{g_{i}}+N_{u}^{g_{i}}$, acorde con las otras definiciones precedentes. Sea $\mu_{i}: G \rightarrow\{0, \ldots, n \times(n-1)\}$ el número de enlaces en todos los senderos que finalizan en $i$, originados por agentes en $N^{i ; g}$ bajo cualquier estrategia conjunta: $\mu_{i}(g)=\mid\left\{(j, k) \in N \times N: g_{j, k}=1, \mathrm{y} \exists l \in N^{i ; g}\right.$ y $\left.l \stackrel{g}{\rightarrow} i \operatorname{con} j, k \in l \stackrel{g}{\rightarrow} i\right\} \mid$. Nótese que puede haber más de un sendero desde $j$ hacia $i$.

Ejemplo 2. Supóngase $N=\{1,2,3,4,5\}$ y la estrategia $g=\left\langle g_{1}, g_{2}, g_{3}, g_{4}, g_{5}\right\rangle$ está dada por la Tabla 2 (* indica quién inició la conexión).

Tabla 2- Perfil de estrategias para el Ejemplo 2

\begin{tabular}{|l|c|c|c|c|c|}
\hline Estrategia & 1 & 2 & 3 & 4 & 5 \\
\hline$g_{1}$ & $\mathrm{X}$ & $1^{*}$ & 0 & 0 & $1^{*}$ \\
\hline$g_{2}$ & 0 & $\mathrm{X}$ & $1^{*}$ & 0 & 0 \\
\hline$g_{3}$ & 0 & 0 & $\mathrm{X}$ & $1^{*}$ & $1^{*}$ \\
\hline$g_{4}$ & 0 & 0 & 0 & $\mathrm{X}$ & 0 \\
\hline$g_{5}$ & 0 & 0 & 0 & 0 & $\mathrm{X}$ \\
\hline
\end{tabular}

${ }^{2}$ Realmente debiera ser $N^{i ; g}=\{k \in N \mid k \stackrel{g}{\rightarrow} i\} \cup\{i\}$. Se incluye $i$ en $N^{i ; g}$ para indicar que $i$ conoce su propia valuación, a pesar del hecho mencionado anteriormente de que $i$ no establece un enlace directo consigo mismo. 
Figura 2 -Red formada por el perfil de estrategias del Ejemplo 2

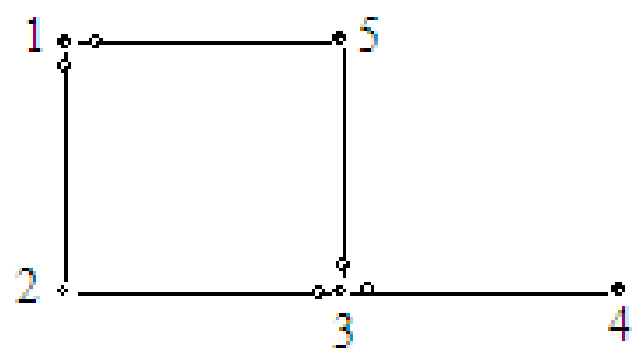

La Figura 2 muestra la red correspondiente. $N^{1 ; \bar{g}}=\{1,2,3,4,5\}, N^{2 ; g}=\{1,2,3,4,5\}$, $N^{3 ; g}=\{1,2,3,4,5\}, N^{4 ; g}=\{1,2,3,4,5\}$ y $N^{5 ; g}=\{1,2,3,4,5\}$. Nótese que bajo flujo de información monodireccional los resultados serían: $N^{1 ; g}=\{1,2,3,4,5\}, \quad N^{2 ; g}=\{2,3,4,5\}$, $N^{3 ; g}=\{3,4,5\}, N^{4 ; g}=\{4\}$ y $N^{5 ; g}=\{5\}$. Esto es, bajo información monodireccional en $g$, el agente 1 accede a la información de todos los agentes mientras que 4 y 5 sólo acceden a su propia información. Ahora, con información bidireccional se tiene que bajo $g$ todos acceden a la información de todos. Los números de enlaces requeridos para obtener la información son $\mu_{1}(g)=5, \mu_{2}(g)=3$ y $\mu_{3}(g)=2$, mientras que $\mu_{4}(g)=\mu_{5}(g)=0$, la misma no cambia para el flujo mono o bidireccional.

\section{II.1 Las conexiones y los beneficios}

Para el presente análisis en particular será fundamental modelar las formas de conexión que prevalecerán en la determinación futura de las funciones de pagos y de costos de cada agente. En el modelo, será crucial distinguir los enlaces directos del agente de sus enlaces indirectos. Los enlaces directos son aquellos enlaces que tienen un sendero de valor uno mientras que los enlaces indirectos son aquellos que tienen un sendero de valor superior a uno, como ya de definió en la sección previamente.

$$
N^{i ; g}=\left\{k \in N \mid g_{i, k}=1\right\}+\left\{h \neq k|h \in N| g_{i, h} \geq 2\right\}
$$

Nuevamente, $N^{i ; g}$ es un vector que recolecta todos aquellos agentes que directa o indirectamente esta conectados con $i$. Aquí también queda discriminado el grado de cercanía de los enlaces. El primer término de (1) se corresponde a los enlaces directos del agente $i$ mientras que el segundo término refiere a los enlaces indirectos del agente, los cuales ya veremos también tiene que solventar. Esto es, los pagos de $i$ son la suma de toda la información que puede ser accedida por ella, menos el costo de los senderos (es decir conexiones directas e indirectas, véase la Figura 3 para una ilustración al respecto con grafos dirigidos con flujo monodireccional) que llegan a ella que están establecidos de acuerdo a $g$ (recuérdese que cada enlace se supone tiene costo unitario). La intuición aquí es que $i$ obtiene un pago por acceder a mayor información pero al mismo tiempo ella tiene que pagar un cargo o arancel por cada uno de los enlaces en los senderos hacia las fuentes de información. Esta forma de financiar las conexiones es, por lo tanto, acumulativa. A medida que la información pasa por más agentes se hace más cara, dado que hay que pagarle a todos los intermediarios. En la Figura 3 se observa como $i$ se conecta directamente con $j_{1} \mathrm{y} j_{2}$, de los que obtiene información directa- 
mente (conjunto de puntos espaciados). Pero también obtiene información indirecta dado que $j_{2}$ esta conectado con $k_{1}$, siendo también que $k_{1}$ se conectó con $k_{2}$ y ésta con $k_{3}$ (conjunto de puntos densos). En definitiva, $i$ accede a la información de los dos conjuntos y debe pagar por los enlaces incluidos dentro de ambos conjuntos. Obsérvese que $j_{3}, j_{4}$ y $k_{4}$ nunca son accedidos por el agente $i$.

Figura 3 -Esquema de conexiones totales e información accedida flujo monodireccional

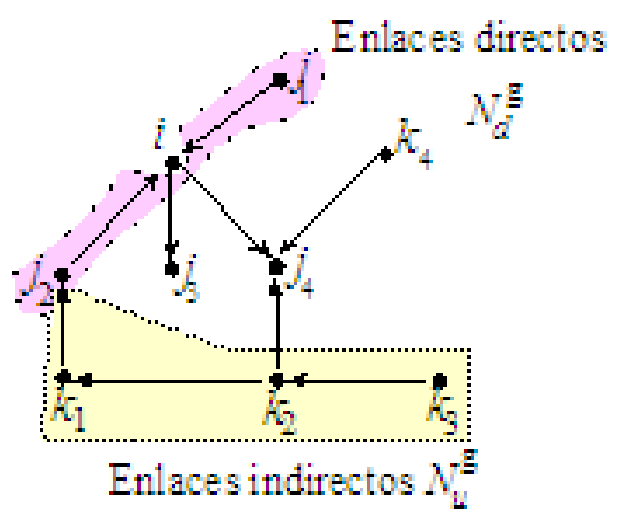

La función de conexiones estipulada anteriormente sigue vigente para este caso pero adquiere una dimensión mayor dado el supuesto de que la información circula en ambos sentidos. Para ello una ilustración gráfica será adecuada. En la Figura 3 se observa la función de conexiones expuesta para el caso monodireccional. En aquel caso, los dos conjuntos accedidos daban cuenta de las estructura de costos y de la información accedida por la agente $i$. Es decir, coincidían los conjuntos punteados en tanto dentro de ellos (las conexiones directas e indirectas) se encontraban los costos que debía abonar la agente $i$ pero también en su interior se hallaban los ingresos por acceder a mayor información. Sin embargo ahora, la estructura de costos se mantiene asociada a los mismos conjuntos punteados del caso monodireccional (Figura 4.a) pero ahora el pago por información es la información poseída por todos los miembros de la red, dado que cada información es accedida sólo con la mera existencia de algún tipo de conexión entre dos agentes sin importan quién inicie el enlace, como se observa en la Figura 4.b.

Figura 4 - Estructura de conexiones e información accedida con flujo bidireccional

(a)

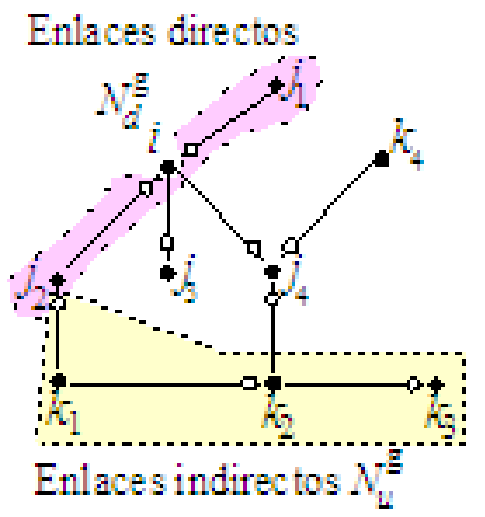

(b)

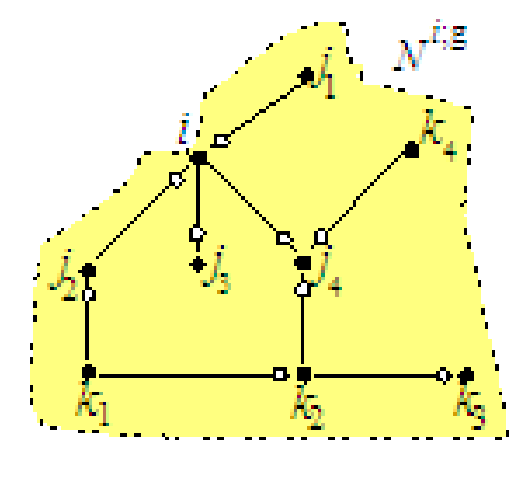


La expresión formal d la función de pagos de cada agente. Ella posee una información propia, $v_{i}$, pero puede acceder a poseer más información si crea una función de conexiones y accede a las informaciones propias y obtenidas por los otros agentes. Por ello, formalizando el valor de la información total accedida por $i, I_{i}$, nos queda:

$$
I_{i}(g)=v_{i}+\sum_{j \neq i \mid j \in \mathrm{g}} I_{j}\left(N^{i ; \mathrm{g}}\right)
$$

donde $I_{j}$ es el valor de la información del agente $j$ que es accedido por $i$ a través del perfil de estrategias g la que se representa por $N^{i ; g}$. Ello determina la estructura de conexión del agente $i$ y la información diversificada a la que accede.

Para hacer a este esquema un juego tenemos que definir los beneficios de los agentes. Supondremos $\Pi_{i}: G \rightarrow R$, la función de beneficios para el agente $i$, que será:

$$
\Pi_{i}(g) \equiv I_{i}(g)-\sum_{j \in N^{i, g}} c \mu_{i}(g)
$$

donde $I_{i}$ representa la información poseída y accedida por el agente $i$ según la estrategia de conexiones $g$ de la definición (1), $c$ representa el costo de cada conexión, que para nuestro caso particular suponemos como $c=1 \mathrm{y} \mu_{i}(\mathrm{~g})$, tal como se definió en la sección anterior, la cantidad de enlaces directos e indirectos que debe solventar la agente $i$.

Considérese ahora una proposición importante:

Proposición 1. Dadas dos estrategias conjuntas $g$ y $g^{\prime}, \Pi_{i}(g) \geq \Pi_{i}\left(g^{\prime}\right)$ sí y sólo sí los correspondientes grafos $N^{i ; g}$ y $N^{i ; g^{\prime}}$ son tales que:

$$
I_{i}(g)-I_{i}\left(g^{\prime}\right) \geq \mu_{i}(g)-\mu_{i}\left(g^{\prime}\right)
$$

Prueba: Trivial.

Este resultado ayuda a comprender la intuición de que el objetivo del agente racional es el de obtener tanta información como sea posible atravesando la menor cantidad de enlaces posibles. Dos casos son de particular interés:

$-\sum_{j \in N^{i: g}} I_{j}=\sum_{j \in N^{i: s^{\prime}}} I_{j}$ y $\mu_{i}(g) \leq \mu_{i}\left(g^{\prime}\right)$,

- $\sum_{j \in N^{i: g}} I_{j} \geq \sum_{j \in N^{i: g^{\prime}}} \boldsymbol{I}_{j} \mathrm{y} \mu_{i}(g)=\mu_{i}\left(g^{\prime}\right)$.

La primera nos muestra que $\Pi_{i}(g) \geq \Pi_{i}\left(g^{\prime}\right)$ si la información obtenida a través de $g$ es la misma que la obtenida por $g$ ' pero el número de enlaces requeridos es menor en $g$ que en $g^{\prime}$. El segundo caso muestra que $\Pi_{i}(g) \geq \Pi_{i}\left(g^{\prime}\right)$ si el número de enlaces requeridos para alcanzar la información es la misma en $g$ que en $g$ ' pero el monto de información obtenida en $g$ es mayor que el monto obtenido en $g$ '.

A continuación se considera cuál es la situación de equilibrio que los agentes enfren$\tan$ ahora. 


\section{EQUILIBRIO Y OPTIMALIDAD}

Dada una red $g \in G$, sea $g_{-i}$ el grafo dirigido obtenido cuando removemos todos los enlaces directos del agente $i$. Entonces, $g$ puede ser escrito como $g=\left(g_{i}, g_{-i}\right)$, significando que $g$ esta formada por la unión de los enlaces de $g_{i}$ y aquellos de $g_{-i}$. Una estrategia $g_{i}$ se dice mejor respuesta del agente $i$ a $g_{-i}$ si

$$
\Pi_{i}\left(g_{i}, g_{-i}\right) \geq \Pi_{i}\left(g_{i}^{\prime}, g_{-i}\right)
$$

para todo $g_{i}^{\prime} \in G_{i}$.

El conjunto de mejores respuestas a $g_{-i}$ es $M R_{i}\left(g_{-i}\right)$. Una red $g=\left(g_{1}, \ldots, g_{n}\right)$ se dice una red de Nash si para cada $i, g_{i} \in M R_{i}\left(g_{-i}\right)$, es decir $g$ (como una estrategia conjunta) es un equilibrio de Nash. Para determinar la estructura de las redes de Nash daremos algunas definiciones que nos permitirán describir propiedades adicionales de las redes.

Dada una red $g$, un conjunto $C \subset N$ es llamado componente de $g$ si para cada par de agentes $i$ y $j$ en $C(i \neq j)$ tenemos que $j \in N^{i, g}$ y no existe un $C^{\prime}, C \subset C^{\prime}$, para el cual esto es cierto. Un componente $C$ se dice mínimo si $C$ no es más componente una vez que $g_{i, j}=1$ entre dos agentes $i$ y $j$ en $C$ es cortado, es decir si $g_{i, j}=0$. Vamos a definir también el concepto de nodo o jugador activo y pasivo: si $i$ forma un enlace con $j$ entonces $i$ es un vecino activo de $j$; si, adicionalmente, $j$ no forma un enlace con $i$ entonces $j$ es un vecino pasivo de $i$.

Una red se dice conectada si soporta un único componente. Sí ese único componente es mínimo $g$ se dice mínimamente conectada. Una red no conectada se dice desconectada. Una instancia particular de las redes mínimamente conectadas es la red de línea, $\mathrm{g}^{l}$ de ahora en más, en la cual los agentes pueden ser etiquetados (por medio de una función $l: N \rightarrow N$ ) como $\{l(1), \ldots, l(n)\}$ y $g_{l(1), l(2)}=g_{l(2) l(3)}=\ldots=g_{l(n-1) l(n)}=1$ y no hay otros enlaces. Dado que el flujo es bidireccional puede ocurrir que dentro de la red de línea los agentes inicien las conexiones de muchas diferentes maneras y aún así permitan el flujo de información a lo largo de la misma. Es decir, dentro de la red de línea existen distintos grafos de activación de la conexión secuencial que otorgan pagos diferentes a los agentes. La red de línea con nodos de activación ordenados ${ }^{3}$ mantiene una configuración de conexiones que hacen que el primero se conecte con el segundo, el segundo con el tercero y así hasta completar la secuencia siendo el anteúltimo que se conecta con el último y no hay más conexiones.

Se pueden destacar algunas formas de activación de la red de línea. Por ejemplo. configuraciones alternativas red de línea con nodos activos impares, $\mathrm{g}^{l i}$, es una topología lineal que puede ser representada por

$g_{l(1), l(2)}=g_{l(3), l(2)}=g_{l(3), l(4)}=\ldots=g_{l(n-3), l(n-4)}=g_{l(n-3), l(n-2)}=\ldots=\left\{\begin{array}{l}g_{l(n-1), l(n)}=1 \text { si } n \text { es par } \\ g_{l(n), l(n-1)}=1 \text { si } n \text { es impar }\end{array}\right.$

\footnotetext{
${ }^{3}$ En algunos trabajos aparece mencionada esta topología como red en cadena. Por ejemplo, Johnson y Gilles (2000).
} 
es decir los nodos impares inician un enlace con sus predecesores y sucesores inmediatos, mientras que los pares pasivamente aceptan hasta completar la secuencia de conexión. Finalmente, una estructura análoga es la red de línea con nodos activos pares, glp que es aquella que cumple con la configuración

$g_{l(2), l(1)}=g_{l(2), l(3)}=g_{l(4), l(3)}=\ldots=g_{l(n-3), l(n-2)}=g_{l(n-1), l(n-2)}=\ldots=\left\{\begin{array}{l}g_{l(n), l(n-1)}=1 \text { si } n \text { es par } \\ g_{l(n-1), l(n)}=1 \text { si } n \text { es impar }\end{array}\right.$

es decir que los nodos etiquetados como pares inician las conexiones hacia sus nodos inmediatamente vecinos mientras que el resto de nodos actúan pasivamente (no inician ellos la conexión). La Figura 5 muestra un ejemplo de cinco agentes para cada uno de los casos descriptos en esta sección.

Figura 5 - Diferentes topologías de redes de línea de conexión secuencial

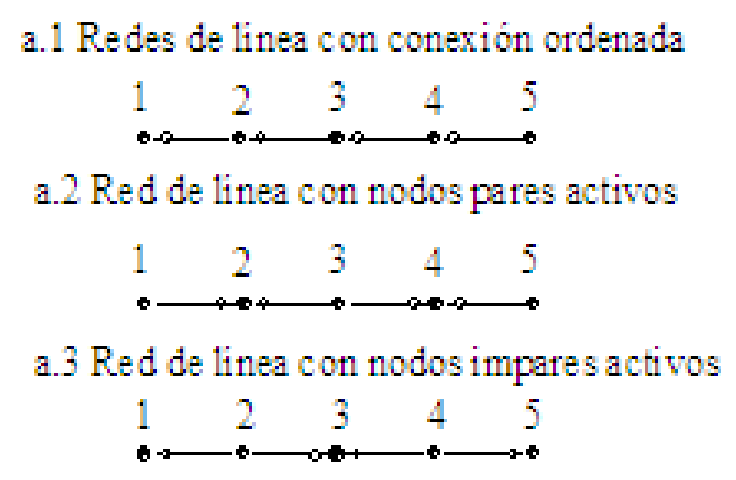

Entonces, con todos estos elementos en la mano y por la Proposición 2, se puede establecer el siguiente resultado (todos los resultados en esta sección corresponden al juego $\langle N, G, \Pi\rangle$, donde $\left.\Pi=\Pi_{1} \times \ldots \times \Pi_{n}\right)$ :

Lema 1: Dada las función de pagos en (4), si $g^{*}$ es una red estricta de Nash, entonces es una red de línea de conexión secuencial con nodos pares o impares activos.

Prueba: Sea $\Pi_{i}: G \rightarrow Z$, para cada $i \in N$ y un equilibrio de Nash estricto $g^{*} \in G$. Entonces, para cada $i$ y cada $g_{i} \in G_{i}$, se tiene que:

$$
\Pi_{i}\left(g_{i}^{*}, g_{-i}^{*}\right)>\Pi_{i}\left(g_{i}, g_{-i}^{*}\right)
$$

Se dice que $g^{*}$ define una red de línea de conexión secuencial. Si es así debe ser cierto para todo agente $i$ que: 


$$
\left\{\begin{array}{l}
\Pi_{i}(g) \equiv \sum_{i \in N} I_{i}-2, \text { si } i \text { es activo, } \\
\Pi_{i}(g) \equiv \sum_{i \in N} I_{i}-1, \text { si } i \text { es el último agente y } \\
\Pi_{i}(g) \equiv \sum_{i \in N} I_{i}, \quad \text { si } i \text { es pasivo. }
\end{array}\right.
$$

En palabras: el máximo de información que puede ser alcanzado en una red de línea de conexión no secuencial es la suma de todas las informaciones poseídas por todos los agentes mientras que el número de enlaces que permitirían hacer disponible la información a cualquiera de ellos es estrictamente positiva pero baja para los nodos activos, casi la mitad de los conectados, y nula para los nodos pasivos (la otra casi mitad de los conectados). Éste se observa como uno de los resultados más eficiente en términos sociales mientras que individualmente resulta también el corolario óptimo.

Sea $n$ par. Se supone por contradicción que $g^{*}$ no es una red de línea de conexión secuencial $^{4}$. Esto significa que para al menos un agente $i$,

$$
\left\{\begin{array}{l}
\Pi_{i}(g) \neq \sum_{i \in N} I_{i}-2, \text { si } i \text { es activo o } \\
\Pi_{i}(g) \neq \sum_{i \in N} I_{i}-1, \text { si } i \text { es el último agente o } . \\
\Pi_{i}(g) \neq \sum_{i \in N} I_{i}, \quad \text { si } i \text { es pasivo. }
\end{array}\right.
$$

1. Considérese primero el caso de un agente activo para quien pudiera existir una estrategia de desvío tal que: $\Pi_{i}\left(g^{*}\right)>\sum_{i \in N} I_{i}-2$. El acceso a mayor información no puede ser mejorado, dado que actualmente $i$ accede a toda la información disponible en la red, por lo que una estrategia que depare mayor beneficio sólo debiera evaluar la disminución del número de conexiones. Si $i$ optará por conectarse con sólo la agente $k+1$ (predecesora) o sólo con $k-1$ (antecesora) anularía la conectividad de la red desconectando en dos subredes a la misma. Con ello se accedería a un nivel de información menor que $\sum_{i \in N} I_{i}$ habiéndose ahorrado sólo una unidad de utilidad en costos de conexión. Por otro lado, si $i$ decidiera conectarse con otras agentes ubicadas en $k \pm j$ tal que $2 \leq j \leq n-j$, entonces dejaría aislados a los agentes intermedios $k \pm k^{\prime}$ con $k^{\prime}$ $<j$. Nuevamente, perdería el acceso a información más valiosa que el beneficio de disminuir los costos de conexión respectivos. Contradicción.

2. Se analiza el caso que $i$ sea el último agente. Si es así, la única manera de desviarse sería cortar su único enlace con el resto de la red. Pero ahorraría una unidad perdiéndolo excepto su propia información. Contradicción.

3. Considérese ahora el caso de un agente $i$, tal que $i$ es pasivo. Veamos si existe una estrategia $g^{*}$ tal que $\Pi_{i}\left(g^{*}\right)>\sum_{i \in N} I_{i}$. Dado que $i$ accede a toda la información incurrir

\footnotetext{
${ }^{4}$ Para la demostración no importa necesariamente si es una red de nodos activos pares o impares dado que con modificaciones triviales se obtiene el mismo resultado.
} 
en costo alguno, no hay acción de $i$ que le permita incrementar el beneficio. Contradicción.

Nótese que no toda red de Nash es linealmente secuencial:

Ejemplo 3. Se tiene que $N=\{1,2,3,4,5\}$ y la estrategia $g=\left\langle g_{1}, g_{2}, g_{3}, g_{4}, g_{5}\right\rangle$ esta dada por la Tabla 3.

Tabla 4 - Perfil de estrategias para el Ejemplo 3

\begin{tabular}{|l|c|c|c|c|c|}
\hline Estrategia & 1 & 2 & 3 & 4 & 5 \\
\hline$g_{1}$ & $\mathrm{X}$ & $1^{*}$ & $1^{*}$ & $1^{*}$ & $1^{*}$ \\
\hline$g_{2}$ & 0 & $\mathrm{X}$ & 0 & 0 & 0 \\
\hline$g_{3}$ & 0 & 0 & $\mathrm{X}$ & 0 & 0 \\
\hline$g_{4}$ & 0 & 0 & 0 & $\mathrm{X}$ & 0 \\
\hline$g_{5}$ & 0 & 0 & 0 & 0 & $\mathrm{X}$ \\
\hline
\end{tabular}

En este caso lo que tenemos es que existe un agente, el 1, que tiene una estrategia $\mathrm{g}_{1}$ que es conectarse con todos los demás mientras que el resto no realiza ninguna conexión. Este tipo de estructura de conexión se denomina red de estrella centralmente sostenida y el grafo de activación de la red se puede observar en la Figura 6.

Figura 6 - Red de estrella centralmente sostenida

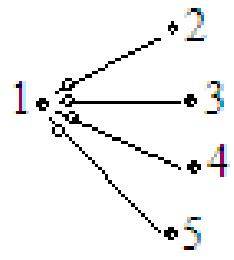

Si se analiza, esta red es una red de Nash. Cualquier desvío implica pérdidas para el agente que incurra en él. Asimismo es una red eficiente en el sentido de Pareto. Sin embargo esta red presenta una asimetría enorme: un solo agente activo sostiene toda la red mientras el resto se comporta pasivamente sin incurrir en costos. El agente central tiene de beneficios $\Pi_{1}(g)=\sum_{i \in N} I_{i}-(n-1)$ mientras que el resto no paga nada y si recibe toda la información.

Cualquier estructura que le brinde la misma cantidad de información a menores costos puede hacer al agente 1 un desvío alentador, acorde con la Proposición 1.

Falk y Kosfeld (2003) encuentran en experimentos de formación de redes que para el caso del modelo de Bala y Goyal (2000), donde los costos no son acumulativos sino que sólo se pagan los costos de enlaces directos, con sujetos de experimento nunca surgía como equilibrio la topología de red de estrella centralmente sostenida. Los autores destacan que la alta asimetría de pagos a la que es sometido el agente central, así como un problema más grande coordinación general, hace inviable que algún sujeto de experimento asuma dicho rol. Ber- 
ninghaus y otros (2004) definen a esta conducta como aversión a la desigualdad, cuya modelación exige considerar ciertos aspectos cualitativos a la hora de formar enlaces. Ellos consideran que los agentes activos poseen mayores privilegios de información que los pasivos (es decir, quien crea un enlace accede al agente y su red de contactos mientras que quien se deja acceder solo accede al agente). De ese modo, los autores logran quebrar la falta de surgimiento de una estructura de equilibrio en experimentos con flujo bidireccional de información, apareciendo en este caso la red periféricamente sostenida como la red equilibrio ${ }^{5}$. Esa línea de explicación es la que sustenta descartar dicha topología como equilibrio también en nuestro modelo. Por ello postulamos lo siguiente;

Proposición 2. $g^{*}$ es una red de línea de conexión secuencial con nodos activos pares o impares sí, y sólo sí, es una red estricta de Nash con el mínimo número de enlaces.

Prueba: Sí $g^{*}$ es una red de línea de conexión secuencial, con grafo de activación con nodos pares y $n$ par. Para cada $i$ el pago está dado por (4). Supongamos que no es red de Nash. Esto es, por al menos un agente $i$, existe una desviación $g^{\prime} \in G$ tal que

$$
\left\{\begin{array}{l}
\Pi_{i}\left(g^{\prime}\right) \neq \sum_{i \in N} I_{i}-2, \text { si } i \text { es activo o } \\
\Pi_{i}\left(g^{\prime}\right) \neq \sum_{i \in N} I_{i}-1, \text { si } i \text { es el último agente } \\
\Pi_{i}\left(g^{\prime}\right) \neq \sum_{i \in N} I_{i}, \text { si } i \text { es pasivo. }
\end{array}\right.
$$

Pero la única manera de alcanzar esto es reduciendo el número de enlaces en los senderos que llevan información de todos los otros agentes hacia $i$. Si $i$ es pasivo no hay estrategia que otorgue mejor pago. Si $i$ es activo puede reducir en uno o en dos los enlaces. Dado que $g^{*}$ es una red de línea de conexión no secuencial, existe sólo un agente $j$ tal que $g_{i, j}^{*}=1$. Hay tres posibilidades de desviación para $g_{i}$ :

- Supóngase que $i$ decide cortar su enlace con la agente $j+1$ pero mantiene su conexión con $j-1$. Con ello ahorra una conexión pero pierde su conexión con los agentes $(j+2 \ldots n)$. Ello deriva en que $\sum_{i=1}^{j-1} I_{i}-1<\sum_{i=1}^{n} I_{i}-2$. Contradicción.

- Si $i$ decide cortar su enlace con la agente $j+1$ y establecer, a su vez, un enlace con la agente $j+2$, manteniendo ese enlace con $j-1$. Entonces $i$ deja de acceder a la información suministrada por la agente $j+1$. Pero por definición $v_{j+1}>c$, por lo que obtendría mayor pago manteniéndose conectado, 0 sea $\left(\sum_{i=1}^{j} I_{i}+\sum_{i=j+2}^{n} I_{i}\right)-2<\sum_{i=1}^{n} I_{i}-2$. Contradicción.

- Finalmente, en el caso que $i$ opta por desconectarse y reducir sus costos de conexión a cero. Pero así sólo accede a su propia información y no deja de acceder a la información de toda la red. Contradicción.

\footnotetext{
${ }^{5}$ La red periféricamente sostenida es la inversa de la red centralmente sostenida. En esta, todos los agentes menos el propio agente central inician enlaces únicamente con el agente central. La asimetría comparada con el caso anterior, se reduce notablemente, dado que hay ahora $n-1$ agentes activos y sólo uno pasivo.
} 
Si $g^{*}$ es un equilibrio de Nash con el mínimo número de enlaces entonces constituye un único componente que incluye todos los agentes en $N$ (de otro modo la información de los agentes que no son accedidos se perderá para al menos algún otro agente, mientras que la reducción en los costos de los enlaces no será lo suficiente como para compensar dicha pérdida. Recuérdese que cada $I_{i}$ es mayor que el costo de un enlace). Como se demostró anteriormente el número mínimo de enlaces que permite estar conectados a todos los agentes es $n$.

\section{III.4 Bienestar social}

De acuerdo al Lema 1 y la Proposición 2, un resultado estable en la interacción estratégica de agentes es la red lineal secuencial con nodos activos pares o impares. Puede afirmarse que ésta es estable porque no hay incentivos, una vez que la estructura de la red circular surge, para cortar los enlaces o formas nuevos, porque la nueva configuración pudiera no otorgar los mismos pagos a los agentes. El argumento hace surgir la pregunta sobre la optimalidad del resultado. Esto es, ¿hay otra configuración que pueda asegurar mejores pagos a los agentes? Antes de responder negativamente esta pregunta, introduciremos dos nociones diferentes de optimalidad que pudiera ser valioso considerar. Una representa la noción de bienestar social asegurada por la red. Formalmente, sea $W: G \rightarrow Z$ definida como $W(g)=\sum_{i=1}^{n} \Pi_{i}(g)$ para $g \in G$. Una red se dice eficiente si $W(g) \geq W\left(g^{\prime}\right)$ para todo $g^{\prime} \in G$.

Por otro lado, tenemos la noción de optimalidad de Pareto. Una red $g$ se dice óptima de Pareto si no existe otra red $g^{\prime}$ tal que para cada $i \in N, \Pi_{i}\left(g^{\prime}\right) \geq \Pi_{i}(g)$ y para al menos un $i, \Pi_{i}\left(g^{\prime}\right)>\Pi_{i}(g)$.

Se llega entonces el siguiente resultado:

Proposición 3. Una red de línea secuencial con grafo de activación de nodos de pares o impares que es estricta de Nash es tanto eficiente como óptima de Pareto.

Prueba. Recuérdese que una red estricta de Nash $g^{*}$ sostiene el máximo pago para cada agente, $\Pi_{i}\left(g^{*}\right)=\sum_{i \in N} I_{i}(g)-2$ si $i$ es activo, $\Pi_{i}\left(g^{*}\right)=\sum_{i \in N} I_{i}(g)-1$ si $i$ es el último conectado y $\Pi_{i}\left(g^{*}\right)=\sum_{i \in N} I_{i}(g)$ si $i$ es pasivo. Entonces, $\Pi_{i}\left(g^{*}\right) \geq \Pi_{i}(g)$ para cada $i \in N$ y cada $g \in G$. De este modo, $g^{*}$ es óptima en el sentido de Pareto. Con el mismo razonamiento, $W\left(g^{*}\right)=\sum_{i \in N} \Pi_{i}\left(g^{*}\right) \geq \sum_{i \in N} \Pi_{i}(g)=W(g)$, para cada $g \in G$. Esto es, $g^{*}$ es eficiente. $\square$

Puede observarse la Tabla 5, a continuación, donde se calcula el beneficio social neto de diferentes configuraciones de red bajo información bidireccional. Todas las redes lineales en secuencia comparten el máximo beneficio junto con la red de estrella centralmente sostenida. Sin embargo como ya se mencionó, dicha configuración adolece de un criterio de simetría 
que si poseen las configuraciones lineales por lo que creemos que será la configuración adoptada por los agentes.

Tabla 5 - Cálculo de pagos por topología

\begin{tabular}{|c|c|c|}
\hline Topología & \multicolumn{2}{|l|}{ Beneficio social neto $\left(\Pi_{i}\right)$} \\
\hline $\begin{array}{l}\text { 1) Red de estrella } \\
\text { centralmente sosteni- } \\
\text { da }\end{array}$ & $\underbrace{\left(\sum_{i \in g} I_{i}-(n-1)\right)}_{\text {Centro de estrella }}+\underbrace{(n-1) \sum_{i \in g} I_{i}}_{\text {Nodos periféricos }}=$ & $n\left(\sum_{i \in g} I_{i}-1\right)+1$ \\
\hline \multicolumn{3}{|c|}{ 2) Redes de línea secuencial } \\
\hline $\begin{array}{l}\text { 2.a) Red de línea con } \\
\text { nodos de activación } \\
\text { ordenados }\end{array}$ & $\sum_{i=1}^{n} I_{i}-(n-i)=$ & $\frac{1}{2} n\left(2 \sum_{i=1}^{n} I_{i}+1\right)-\frac{1}{2} n^{2}$ \\
\hline \multirow{2}{*}{$\begin{array}{l}\text { 2.a) Red de línea con } \\
\text { nodos de activación } \\
\text { impares }\end{array}$} & $\begin{array}{l}n \text { par : } \\
\underbrace{\left(\sum_{i \in g} I_{i}-1\right)}_{\text {Nodo inicial }}+\underbrace{\left(\frac{n}{2}-1\right)\left(\sum_{i \in g} I_{i}-2\right)}_{\text {Resto de nodos impares }}+\underbrace{\frac{n}{2}\left(\sum_{i \in g} I_{i}\right)}_{\text {Nodos pares }}+=\end{array}$ & $n\left(\sum_{i \in g} I_{i}-1\right)+1$ \\
\hline & $\begin{array}{l}n \text { impar: } \\
(\underbrace{\left(\sum_{i \in g} I_{i}-1\right)}_{\text {Nodo inicial }}+\underbrace{\left(\frac{n}{2}-1\right)\left(\sum_{i \in g} I_{i}-2\right)}_{\text {Resto de nodos impares }}+\underbrace{\frac{n}{2}\left(\sum_{i \in g} I_{i}\right)}_{\text {Nodos pares }}+=\end{array}$ & $n\left(\sum_{i \in g} I_{i}-1\right)+1$ \\
\hline \multirow{2}{*}{$\begin{array}{l}\text { 3.b) Red de línea con } \\
\text { nodos de activación } \\
\text { pares }\end{array}$} & $\begin{array}{l}n \text { par: } \\
\underbrace{\left(\frac{n}{2}-1\right)\left(\sum_{i \in g} I_{i}-2\right)}_{\text {Nodos pares menos nodo final }}+\underbrace{\frac{n}{2}\left(\sum_{i \in g} I_{i}\right)}_{\text {Nodos impares }}+\underbrace{\left(\sum_{i \in g} I_{i}-1\right)}_{\text {Nodo final }}=\end{array}$ & $n\left(\sum_{i \in g} I_{i}-1\right)+1$ \\
\hline & $n$ impar: $\underbrace{\left(\frac{n-1}{2}\right)\left(\sum_{i \in g} I_{i}-2\right)}_{\text {Nodos pares }}+\underbrace{\left(\frac{n+1}{2}\right)\left(\sum_{i \in g} I_{i}\right)}_{\text {Nodos impares }}=$ & $n\left(\sum_{i \in g} I_{i}-1\right)+1$ \\
\hline
\end{tabular}

\section{CONCLUSION}

Las restricciones que impone a los beneficios la función de costos acumulativos restringe enormemente la factibilidad de encontrar una red amplia como red estricta de Nash. La red óptima no puede expandirse demasiado sin incurrir en crecientes costos que afectan los beneficios de los agentes. Bajo flujo de información bidireccional quedan demarcadas algunas redes de Nash, siendo la menos asimétrica la que los agentes terminarán adoptando. Esta configuración es la red lineal secuencial con nodos de activación pares o impares, donde agentes intermedios realizan conexiones con sus dos vecinos inmediatos cortando la acumulación de costos de la función previamente definida. Dicho resultado resulta, también maximizador del bienestar social. 
Como futuras extensiones al trabajo puede mencionarse la interesante propuesta de Berninghaus y otros (2004), tomada por Goyal (2004), sugiriendo cualificar los enlaces de modo de valorar más la creación de enlaces que mantener una actitud pasiva de dejarse conectar. Esta sugerencia, podría brindar una topología más amplia como solución de equilibrio estricto de Nash para los modelos mono y bidireccional.

\section{BIBLIOGRAFIA}

Berninghaus, S.K., M. Ott y B. Vogt (2004), On networks and 'stars' - Recent results in network experiments, University of Karlsruhe, mimeo.

Bala, V. y Goyal, S. (2000), "A Noncooperative Model of Network Formation". Econometrica, (68), pp. 1181-1229.

Dutta, B., van den Nouweland, A. y Tijs, S. (1998), "Link Formation in Cooperative Situations". International Journal of Game Theory, (27), pp. 245-255.

Falk, A. y Kosfeld, M. (2003), "It's all about connections: Evidence on network formation". IEW Working Paper 21, Universität Zurich.

Goyal, S. (2004), Strong and weak links, Queen Mary University, Londres, mimeo.

Johnson, C.A. y R.P. Gilles (2000), "Spatial Social Networks". Review of Economic Design, (5), pp.273-299.

Jackson, M. y Wolinsky, A. (1996), "A Strategic Model of Social and Economic Networks", Journal of Economic Theory, (71), pp. 44 -74.

Larrosa, J.M.C. y F. Tohmé (2003), "Formación de redes dirigidas circulares con costos de enlace compartidos", Estudios Económicos XX, (41), ene-dic, pp. 27-48.

Qin, C.Z. (1996), "Endogenous Formation of Cooperative Structures”, Journal of Economic Theory 69: 218-226.

Slikker, M., Gilles, R., Norde, H. y Tijs, S. (2002), Directed Networks, Allocation Properties and Hierarchy Formation, Center of Economic Research, University of Tilburg, mimeo.

, M. y van den Nouweland, A. (2001), "A One-Stage Model of Link Formation and Payoff Division”, Games and Economic Behavior,(34), pp. 153-175. 